\title{
Preoperative chlorhexidine mouthwash to reduce pneumonia after cardiac surgery: A systematic review and meta-analysis
}

\author{
Amit Bardia, MBBS, ${ }^{a}$ Daina Blitz, MD, ${ }^{a}$ Feng Dai, MS, PhD, ${ }^{b}$ Denise Hersey, MLS, MA, \\ Sayuri Jinadasa, MD, ${ }^{\mathrm{d}}$ Mayanka Tickoo, MD, ${ }^{\mathrm{e}}$ and Robert B. Schonberger, $\mathrm{MD}^{\mathrm{a}}$
}

\begin{abstract}
Objective: Postoperative pneumonia is one of the most common complications after cardiac surgery, entailing increased patient morbidity, mortality, and health care burden. The primary aim of this study was to assess whether preoperative chlorhexidine mouthwash is associated with reduced postoperative pneumonia after cardiac surgery.
\end{abstract}

Methods: A comprehensive systematic search of NLM Pubmed, Embase, Scopus, and Cumulative Index of Nursing and Allied Health was executed to include the studies since inception to June 27, 2017, which assessed the effects of preoperative chlorhexidine gluconate mouthwash on postoperative pneumonia. Studies were identified by 2 independent reviewers, and data were extracted using a predefined protocol. Random effects models were run to obtain risk ratios with $95 \%$ confidence intervals. Quality of evidence was evaluated using Grading of Recommendations Assessment, Development and Evaluation criteria. Postoperative pneumonia after cardiac surgery was the primary outcome of the study.

Results: Five studies including a cumulative of 2284 patients were included. A total of 1125 patients received preoperative chlorhexidine. Use of chlorhexidine gluconate was associated with reduced risk of postoperative pneumonia compared with the patients who did not receive it (risk ratio, $0.52 ; 95 \%$ confidence interval, $0.39-0.70 ; P<.001)$. No adverse effects from chlorhexidine gluconate mouthwash were reported by any of these studies.

Conclusions: Among the patients receiving preoperative chlorhexidine mouthwash, the risk of postoperative pneumonia is reduced by approximately onehalf; its adoption in preoperative protocols could help improve patient outcomes.

(J Thorac Cardiovasc Surg 2019;158:1094-100)

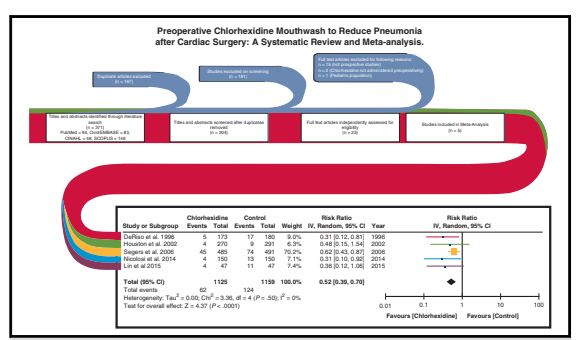

Postoperative pneumonia after cardiac surgery with respect to chlorhexidine mouthwash.

\section{Central Message}

Preoperative chlorhexidine use is associated with a reduction in postoperative pneumonia after cardiac surgery.

\section{Perspective}

The findings of this paper suggest a reduction in postoperative pneumonia after cardiac surgery with the use of preoperative chlorhexidine. Incorporation of this relatively simple maneuver may significantly improve postoperative outcomes in the cardiac surgery patient population.

See Commentary on page 1101.
Postoperative pneumonia is one of the most prevalent hospital-acquired infections after cardiac surgeries, attributing to a 4-fold increase in the risk of mortality. ${ }^{1}$ In addition, it incurs tremendous burden on the health care

\footnotetext{
From the ${ }^{\mathrm{a}}$ Department of Anesthesiology and ${ }^{\mathrm{e}}$ Pulmonary and Critical Care Medicine, Department of Internal Medicine, Yale School of Medicine, New Haven, Conn; ${ }^{b}$ Department of Biostatistics, Yale School of Public Health, New Haven, Conn; ${ }^{\mathrm{c} S}$ Science Libraries, Scholarly Collections and Research Services, Princeton University, Princeton, NJ; and ${ }^{\mathrm{d}}$ Department of Surgery, Beth Israel Deaconess Medical Center, Harvard Medical School, Boston, Mass.

Drs Bardia and Blitz contributed equally to this article.

Received for publication Aug 28, 2018; revisions received Dec 18, 2018; accepted for publication Jan 6, 2019; available ahead of print Feb 28, 2019.

Address for reprints: Amit Bardia, MBBS, Department of Anesthesiology, Yale School of Medicine, New Haven, CT 06515 (E-mail: amit.bardia@yale.edu). 0022-5223/\$36.00

Copyright (c) 2019 by The American Association for Thoracic Surgery https://doi.org/10.1016/j.jtcvs.2019.01.014
}

system, as it directly impacts ventilator days, length of stay in the intensive care unit (ICU), and total hospital length of stay. ${ }^{2}$ Preventing pneumonia after cardiac surgery is especially challenging since the usual preventive maneuvers used in the ICU, ${ }^{3,4}$ such as semirecumbent positioning, subglottic suctioning, and sedation interruption, are not practical in the intraoperative period.

Daily mouthwash with chlorhexidine gluconate, a broadspectrum antimicrobial, has been shown to significantly reduce the incidence of ventilator-associated pneumonia

- Scanning this $\mathrm{QR}$ code will take you to the article title page to access supplementary information. 


\section{Abbreviations and Acronyms \\ $\mathrm{CI}=$ confidence interval \\ ICU $=$ intensive care unit \\ $\mathrm{RCT}=$ randomized controlled trial \\ $\mathrm{RR}=$ risk ratio \\ $\mathrm{VAP}=$ ventilator-associated pneumonia}

(VAP). ${ }^{5,6}$ Despite some evidence signaling its beneficial effect, ${ }^{7}$ routine use of preoperative chlorhexidine mouthwash for preventing postoperative pneumonia after cardiac surgeries is not a universal practice. Data regarding the efficacy and safety of chlorhexidine mouthwash in preventing postoperative pneumonia may help health care providers to improve postoperative outcomes. To address this, we conducted a meta-analysis and systematic review to assess the efficacy of preoperative chlorhexidine gluconate mouthwash in reducing postoperative pneumonia after cardiac surgery.

\section{METHODS}

\section{Data Sources and Searches}

This systematic review is reported according to the Preferred Reporting Items for Systematic Reviews and Meta-analyses statement for meta-analysis. ${ }^{8}$

\section{Search and Selection Strategy}

A comprehensive search using NLM Pubmed, Embase, Scopus, and Cumulative Index of Nursing and Allied Health was performed. The Medical Subject Headings terms and key words are shown in the Online Data Supplement 1. To summarize in brief, the terms used included preoperative, chlorhexidine, mouthwash, cardiac surgery, heart surgery, pneumonia, prevent, and cardiovascular. All searches were run on June 27, 2017, by the institutional librarian. Study inclusion criteria were decided a priori. Only human studies involving cardiac surgeries on adult patient populations that tested the effect of chlorhexidine preoperatively on postoperative pneumonia were included. This included randomized control trials as well as studies using a pre-post intervention design with historical controls. No language restrictions were imposed.

For articles meeting the initial screening, full-text articles were then independently evaluated to assess for eligibility by 2 investigators (D.B. and A.B.). The investigators (D.B. and A.B.) independently used a predefined questionnaire for study evaluation. In the case of discrepancy, this was to be resolved by discussion with the senior author (R.S.). All the outcomes were based on aggregated data that were extracted from results of eligible studies. No individual participant data were available for the meta-analysis. Data were independently extracted by 2 coauthors (A.B. and D.B.) and compared to check for inconsistency/missing data. Data were then analyzed according to our predefined statistical plan.

\section{Outcomes}

The primary outcome was postoperative pneumonia after cardiac surgery. Secondary outcomes included postoperative mortality, grampositive pneumonia, and gram-negative pneumonia in the 2 groups.

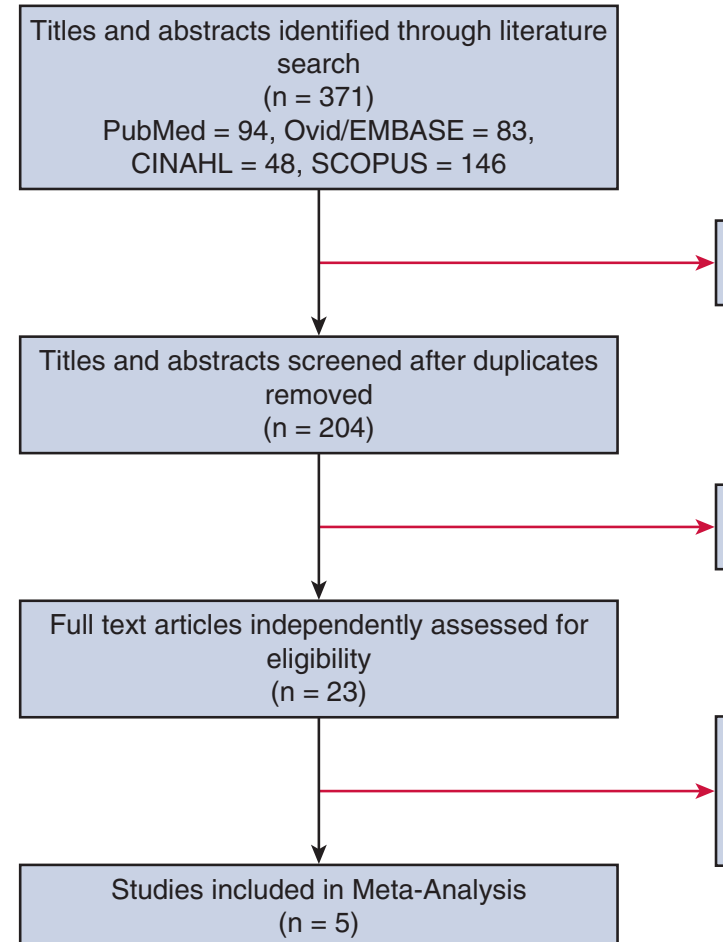

Duplicate articles excluded $(n=167)$

\section{Studies excluded on screening} $(n=181)$

Full text articles excluded for following reasons: $n=15$ (not prospective studies)

$\mathrm{n}=2$ (Chlorhexidine not administered preoperatively) $\mathrm{n}=1$ (Pediatric population)

FIGURE 1. Preferred Reporting Items for Systematic Reviews and Meta-analyses flow diagram depicting study inclusion process. NLM Pubmed, Embase, Scopus, and Cumulative Index of Nursing and Allied Health $(C I N A H L)$ databases were searched for key terms related to preoperative chlorhexidine mouthwash, postoperative pneumonia, and cardiac surgery. Five studies were included in the metanalysis after removal of duplicates and studies that did not meet the inclusion criteria. 


\section{Quality of Evidence}

The quality of evidence was audited using the Grading of Recommendations Assessment, Development and Evaluation criteria for meta-analysis. ${ }^{9}$

\section{Statistical Analysis}

Statistical analysis and reporting were performed based on previous published guidelines by the journal. ${ }^{10-12}$ Random-effects model method was implemented in Review Manager (RevMan) software version 5.3 (The Nordic Cochrane Centre, The Cochrane Collaboration, Copenhagen, Denmark, 2014). Risk ratio (RR) and $95 \%$ confidence intervals (CIs) were used for reporting the magnitude of the effect size for binary outcomes. The $\mathrm{I}^{2}$ statistic was used to assess heterogeneity, and Cochrane's Q test statistic was used to assess its statistical significance. All $P$ values $<.05$ were considered to be statistically significant. Sensitivity analysis was also performed to include only data from randomized controlled trials (RCTs). The limited number of studies precluded the use of a funnel plot or metaregression analyses.

\section{RESULTS \\ Study Characteristics}

Of the 371 citations, after assessment of full-text articles and adjusting for duplicates, 5 articles met the inclusion criteria. ${ }^{13-17}$ The Preferred Reporting Items for Systematic Reviews and Meta-analyses flow diagram of the study selection process is shown in Figure 1.

A total of 2284 cumulative subjects $(26.7 \%$ women $)$ from the 5 articles were included in this study, of whom 1125 received preoperative chlorhexidine. Of these, only 1 study (Nicolosi and colleagues) ${ }^{16}$ was a pre-post study, and the remaining 4 studies were RCTs. The key clinical characteristics of these studies are listed in Table 1. ${ }^{13-19}$

The majority of surgeries in all the studies comprised coronary artery bypass grafting, valvular heart surgery, or both. None of the studies included ventricular assist device

TABLE 1. Clinical characteristics of the included studies

\begin{tabular}{|c|c|c|c|c|c|}
\hline Study (y) & Location & $\begin{array}{l}\text { Type of cardiac } \\
\text { surgery }\end{array}$ & $\begin{array}{c}\text { Sample size } \\
\text { (chlorhexidine vs } \\
\text { control) }\end{array}$ & $\begin{array}{c}\text { Chlorhexidine } \\
\text { regimen }\end{array}$ & $\begin{array}{l}\text { Criteria for } \\
\text { diagnosing } \\
\text { pneumonia }\end{array}$ \\
\hline Lin et al, $2015^{15}$ & China & All cardiac surgeries & 94 (47 vs 47) & $\begin{array}{l}\text { Chlorhexidine vs } \\
\text { normal saline }\end{array}$ & $\begin{array}{l}\text { Clinical Pulmonary } \\
\text { Infection Score } \\
(\mathrm{CPIS}) \text { criteria }^{18}\end{array}$ \\
\hline Nicolosi et al, 2014 ${ }^{16}$ & Argentina & $\begin{array}{l}\text { Nonemergent cardiac } \\
\text { surgeries }\end{array}$ & 300 (150 vs 150$)$ & $\begin{array}{l}\text { Chlorhexidine vs } \\
\text { usual care }\end{array}$ & $\begin{array}{l}\text { Evidence of a new } \\
\text { lung infiltrate on } \\
\text { chest radiograph } \\
\text { and at least } 2 \text { of the } \\
\text { following: } \\
\text { leukocytosis, fever, } \\
\text { or purulent } \\
\text { tracheobronchial } \\
\text { secretion }\end{array}$ \\
\hline Segers et al, $2006^{17}$ & The Netherlands & $\begin{array}{l}\text { Nonemergent cardiac } \\
\text { surgeries }\end{array}$ & 991 (500 vs 491) & $\begin{array}{l}\text { Chlorhexidine vs } \\
\text { placebo of } \\
\text { comparable color, } \\
\text { taste, and smell }\end{array}$ & CDC criteria $^{19}$ \\
\hline Houston et al, $2002^{14}$ & US & $\begin{array}{l}\text { CABG, valvular } \\
\text { surgery, combined } \\
\text { CABG and valvular } \\
\text { surgery }\end{array}$ & 561 (270 vs 291$)$ & $\begin{array}{l}\text { Chlorhexidine vs } \\
\text { Listerine (McNeil } \\
\text { Consumer } \\
\text { Healthcare, Fort } \\
\text { Washington, Penn) }\end{array}$ & $\begin{array}{l}\text { Nature of } \\
\text { tracheobronchial } \\
\text { secretions, evidence } \\
\text { of fever, degree of } \\
\text { leukocytosis, } \\
\text { microbial culture } \\
\text { results, and } \\
\text { evidence of } \\
\text { pulmonary infiltrate }\end{array}$ \\
\hline DeRiso et al, $1996^{13}$ & US & $\begin{array}{l}\text { CABG, valvular } \\
\text { surgery, combined } \\
\text { CABG and valvular } \\
\text { surgery, septal } \\
\text { surgery, cardiac } \\
\text { tumor excision }\end{array}$ & 353 (173 vs 180$)$ & $\begin{array}{l}\text { Chlorhexidine vs base } \\
\text { solution of } \\
\text { chlorhexidine }\end{array}$ & $\begin{array}{l}\text { New or progressing } \\
\text { pulmonary } \\
\text { infiltrate, fever, } \\
\text { leukocytosis, and } \\
\text { purulent } \\
\text { tracheobronchial } \\
\text { secretions }\end{array}$ \\
\hline
\end{tabular}




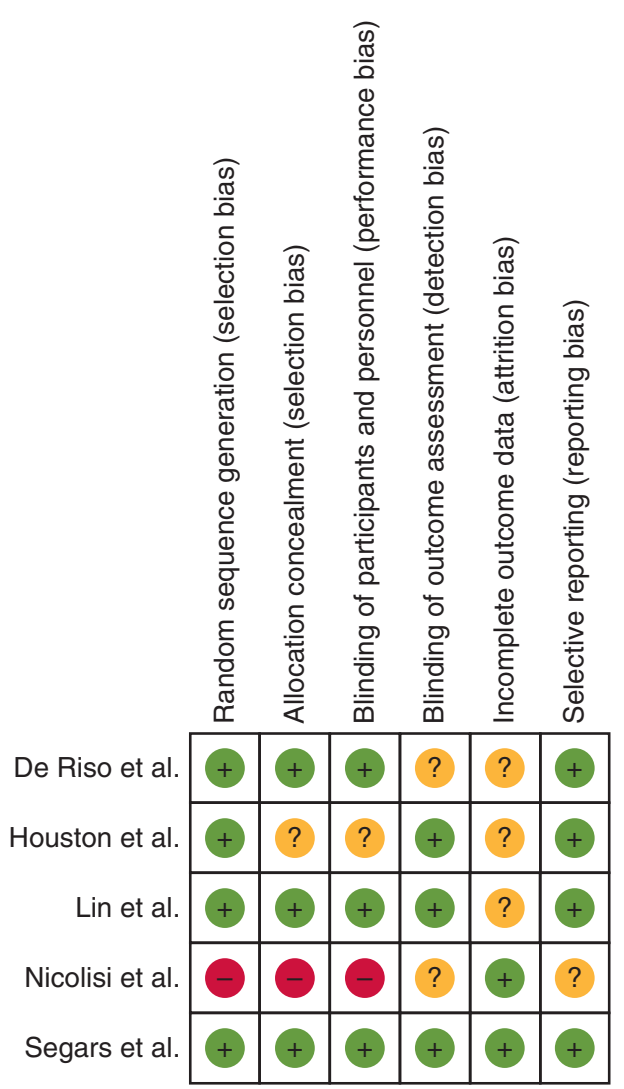

FIGURE 2. Risk of bias summary for the included clinical studies. All the 5 studies were assessed for risk of bias based on Cochrane's Collaboration tool. Green, yellow, and red colors signify low, unclear, and high risk of bias, respectively.

placement, and 1 study included 5 heart transplant surgeries (Lin and colleagues $^{15}$ ). Common exclusion criteria included preoperative infection, preoperative intubation, pregnancy, emergency surgery, and intraoperative death. Formulations containing $0.12 \%$ to $0.2 \%$ chlorhexidine were used for preoperative decontamination by the various study groups. The methodology used for oral decontamination ranged from one preoperative rinse (DeRiso and colleagues) ${ }^{13}$ to rinses every 12 hours for 3 days preoperatively (Nicolisi and colleagues). ${ }^{16}$ Perioperative antimicrobial coverage by cephalosporins or vancomycin was used by all the investigators (except Lin and colleagues, ${ }^{15}$ who did not mention perioperative antibiotic coverage). Standard guidelines for diagnosing pneumonia were used by all the study groups, which included new or progressing pulmonary infiltrates on chest radiograph, fever, leukocytosis, purulent tracheobronchial secretions, and microbial culture results.

\section{Risk of Bias Assessment}

The Cochrane risk of bias assessment tool was used for assessment of the trials (Figure 2). ${ }^{9,20}$ Overall, 4 trials $(80 \%)$ had adequate sequence generation and $3(60 \%)$ had allocation concealment. Adequate blinding of study personnel including outcome assessors was present in 3 $(60 \%)$ of the trials. Two of the 5 studies were considered to be at a low risk for incomplete outcomes data. In the cumulative assessment of bias, 3 of the 5 trials were deemed to have an overall low risk of bias.

\section{Primary Outcome}

Use of chlorhexidine was significantly associated with reduced risk of postoperative pneumonia (RR, 0.52; $95 \%$ CI, 0.39-0.70; $P<.001$ ) (Figure 3). There was minimal heterogeneity among the reported trials, $\mathrm{I}^{2}=0 \%$. We also performed a sensitivity analysis for the primary outcome by including only RCTs. No significant change in the effect or the overall outcome was observed (RR, $0.54 ; 95 \% \mathrm{CI}$, $0.40-0.73 ; P<.0001)$.

\section{Secondary Outcomes}

Postoperative pneumonia in the chlorhexidine group was significantly lower for gram-positive organisms (RR, 0.30; $95 \% \mathrm{CI}, 0.12-0.76 ; P=.01 ; \mathrm{I}^{2}=0 \%$ ) (Figure 4). The association did not reach statistical significance in the gramnegative group (RR, $0.47 ; 95 \% \mathrm{CI}, 0.22-1.02 ; P=.06$; $\mathrm{I}^{2}=56 \%$ ). No significant difference in the in-hospital

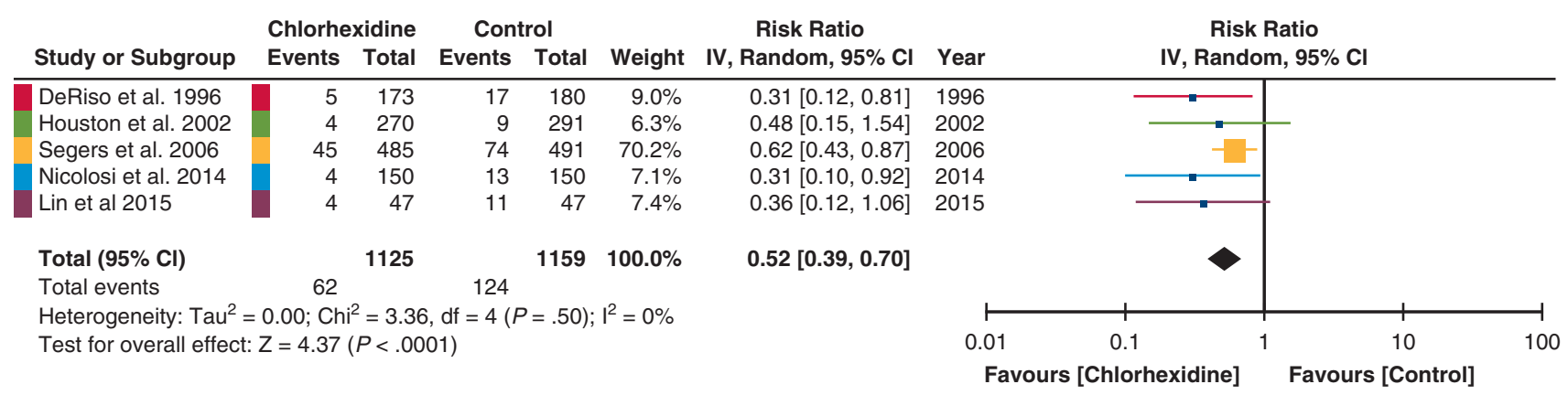

FIGURE 3. Forest plot comparing postoperative pneumonia after cardiac surgery in patients with or without preoperative chlorhexidine mouthwash. Use of chlorhexidine was significantly associated with reduced risk of postoperative pneumonia (risk ratio, $0.52 ; 95 \% \mathrm{CI}, 0.39-0.70 ; P<.001$ ). IV, Inverse variance weighting; $C I$, confidence interval. 
Gram Positive Pneumonia

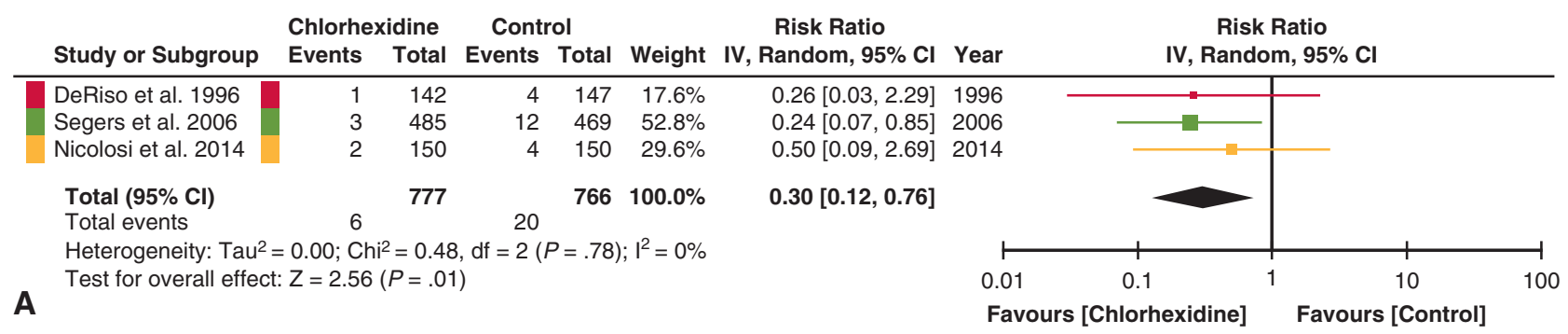

Gram Negative Pneumonia

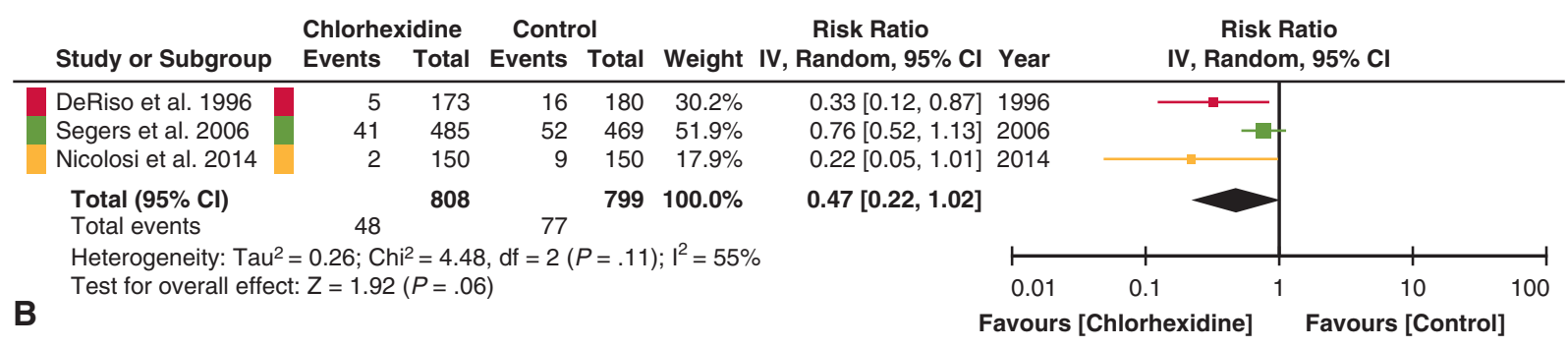

FIGURE 4. Forest plot comparing postoperative pneumonia with gram-positive organisms (A) and gram-negative organisms (B) after cardiac surgery in patients with or without preoperative chlorhexidine mouthwash. Postoperative pneumonia in the chlorhexidine group was significantly lower for grampositive organisms (risk ratio, $0.30 ; 95 \% \mathrm{CI}, 0.12-0.76 ; P=.01 ; \mathrm{I}^{2}=0 \%$ ). The association did not reach statistical significance in the gram-negative group (risk ratio, $0.47 ; 95 \% \mathrm{CI}, 0.22-1.02 ; P=.06 ; \mathrm{I}^{2}=55 \%$ ). $I V$, Inverse variance weighting; $C I$, confidence interval.

mortality rates was observed (RR, $0.98 ; 95 \% \mathrm{CI}, 0.43-2.23$; $P=.95 ; \mathrm{I}^{2}=47 \%$ ) (Figure 5). Other parameters such as duration of mechanical ventilation, length of ICU stay, and length of hospital stay were not uniformly reported by all the studies (Online Data Supplement 2). The studies by Nicolosi and colleagues and Segers and colleagues report a small benefit in hospital stay with chlorhexidine mouthwash use, whereas DeRiso and colleagues report no such benefit. The studies report no significant differences in ventilator time between the 2 groups. None of the studies reported any adverse effects from chlorhexidine mouthwash.

\section{DISCUSSION}

In this systemic review and meta-analysis, direct evidence from 5 studies in 2284 patients was combined to assess the effect of preoperative chlorhexidine gluconate mouthwash on postoperative pneumonia after cardiac surgeries. The study has several important findings. First, postoperative pneumonia is a common postoperative complication after cardiac surgery, with a cumulative incidence of about $8 \%$ among all studies combined. Second, chlorhexidine mouthwash was associated with significant reduction in the risk of postoperative pneumonia after cardiac surgery. Third, this protective effect is particularly profound for gram-positive organisms.

Chlorhexidine mouthwash to prevent VAP in the ICU setting has been recognized. ${ }^{21-23}$ The findings of our study resemble those by Hua and colleagues. ${ }^{24}$ In their metanalysis consisting of 6016 participants (37 RCTs) who were critically ill and expected to require mechanical ventilation for a minimum of 48 hours, use of chlorhexidine mouthwash was found to be associated with a decreased

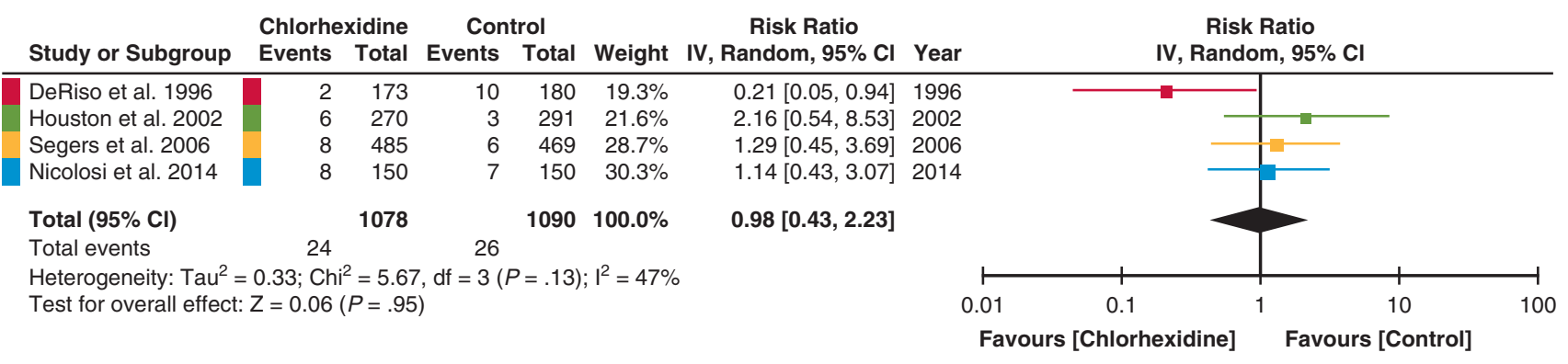

FIGURE 5. Forest plot comparing postoperative in-hospital mortality after cardiac surgery with respect to chlorhexidine mouthwash. No significant difference in the in-hospital mortality rates was observed (risk ratio, $0.98 ; 95 \% \mathrm{CI}, 0.43-2.23 ; P=.95 ; \mathrm{I}^{2}=47 \%$ ). $I V$, Inverse variance weighting; $C I$, confidence interval. 


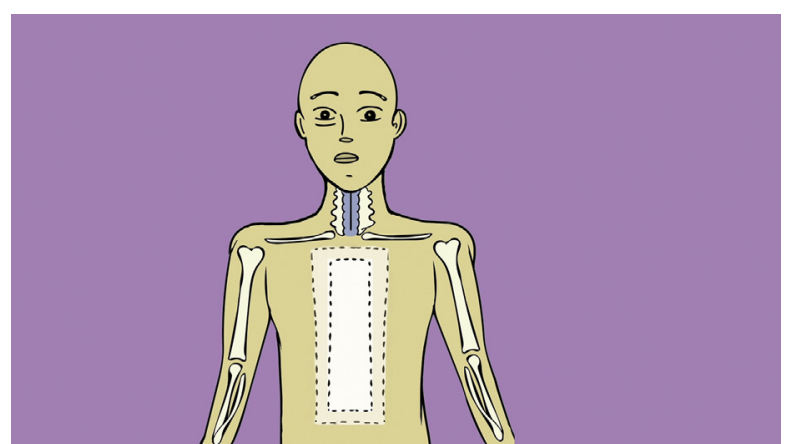

VIDEO 1. Video abstract summarizing the systematic review and metaanalysis of the use of preoperative chlorhexidine mouthwash to prevent postoperative pneumonia after cardiac surgery. Video available at: https://www.jtcvs.org/article/S0022-5223(19)30066-2/fulltext.

rate of VAPs (RR, 0.75; 95\% CI, 0.62-0.91; $P=.004$; $\left.\mathrm{I}^{2}=35 \%\right)$. Similar to our findings, there was no mortality benefit with chlorhexidine mouthwash (RR, 1.09; 95\% CI, $\left.0.96-1.23 ; P=.20 ; \mathrm{I}^{2}=0 \%\right)$. However, the role of preoperative chlorhexidine in preventing postoperative pneumonia remains unclear. Our study highlights the protective effect of preoperative chlorhexidine mouthwash for preventing postoperative pneumonia after cardiac surgery.

Microaspiration of microflora in the aero-digestive tract has been implicated for the pathophysiology of pneumonia in intubated patients. Oral decontamination by chlorhexidine could possibly decrease this microbial burden and hence exert its protective effect. This putative biological basis for the protective effect of chlorhexidine was consistent with the finding that its efficacy appears to be particularly pronounced for preventing gram-positive pneumonia. The predominant bacteria in this group are Staphylococcus species, which are known to frequently colonize the nasopharyngeal region. Chlorhexidine mouthwash thus has a highly plausible biological basis to decrease the incidence of postoperative pneumonia. The fact that a similar effect was not seen for gram-negative organisms would be consistent with the notion that a majority of gram-negative pneumonia infections are likely secondary to aspiration of gastrointestinal contents in intubated patients, and it would not be expected that mouthwash by chlorhexidine in isolation would provide protection from the gastrointestinal microbiome.

Importantly, we did not observe a significant decrease in overall in hospital mortality. A possible reason for this could be that in-hospital mortality occurs due to multiple etiologies after cardiac surgery, with postoperative pneumonia being an important factor in only a minority of such cases. Another possible reason could be that our study was underpowered to detect a difference, since in-hospital mortality was an infrequent outcome $(2.3 \%$ among studies that reported the outcome).
Our study has some limitations. First, as only a few studies met the inclusion criteria, the small sample size may influence the generalizability of these results to the wider cardiac surgery population. Because of the small number of studies, meta-regression analysis could also not be performed. It is also important to note that the low value of $\mathrm{I}^{2}$ statistic could be due to a small sample size. However, we reported $95 \%$ CIs in addition to point estimates for additional characterization of the heterogenicity and chose a random effects model that would be able to accommodate differential effects of the intervention among studies.

Second, our study only examined cardiac surgeries, and thus its generalizability to noncardiac surgical populations is unclear. The studies used differing protocols for preoperative chlorhexidine mouthwash. It is difficult to comment whether a particular protocol was superior to another. Similarly, the control group did not have exactly the same exposure. This may be a source of heterogeneity of effects, further justifying our choice of a random effects model to accommodate the potential for heterogeneity. Finally, although the study was not registered in PROSPERO, it was performed according to an a priori statistical plan as mentioned previously.

\section{CONCLUSIONS}

Preoperative chlorhexidine is associated with a significant reduction in postoperative pneumonia after cardiac surgery. This relatively simple intervention was not associated with any adverse events in the studies included in the metaanalysis. Preoperative chlorhexidine mouthwash should be considered in suitable patients undergoing cardiac surgeries (Video 1).

\section{Conflicts of Interest}

Dr Schonberger holds an equity position in Johnson \& Johnson. All other authors have nothing to disclose with regard to commercial support.

We thank Suveen Angraal, MD, for his help with the statistical inputs; Dr Manuel Fontes, Dr Chris Szabo, and Dr Arnar Geirsson for the idea conception and departmental support; and Garrett Sendlewski for his extensive help with graphical abstract and video summary creation. This publication was made possible by CTSA Grant Number UL1 TR000142 from the National Center for Advancing Translational Science (NCATS), a component of the National Institutes of Health (NIH). Its contents are solely the responsibility of the authors and do not necessarily represent the official view of NIH.

\section{References}

1. Kinlin LM, Kirchner C, Zhang H, Daley J, Fisman DN. Derivation and validation of a clinical prediction rule for nosocomial pneumonia after coronary artery bypass graft surgery. Clin Infect Dis. 2010;50:493-501.

2. He S, Chen B, Li W, Yan J, Chen L, Wang X, et al. Ventilator-associated pneumonia after cardiac surgery: a meta-analysis and systematic review. J Thorac Cardiovasc Surg. 2014;148:3148-55.e3141-5. 
3. Hellyer TP, Ewan V, Wilson P, Simpson AJ. The intensive care society recommended bundle of interventions for the prevention of ventilator-associated pneumonia. J Intensive Care Soc. 2016;17:238-43.

4. Morris AC, Hay AW, Swann DG, Everingham K, McCulloch C, McNulty J, et al Reducing ventilator-associated pneumonia in intensive care: impact of implementing a care bundle. Crit Care Med. 2011;39:2218-24.

5. Ozcaka O, Basoglu OK, Buduneli N, Tasbakan MS, Bacakoglu F, Kinane DF. Chlorhexidine decreases the risk of ventilator-associated pneumonia in intensive care unit patients: a randomized clinical trial. J Periodontal Res. 2012;47:584-92.

6. Fourrier F, Cau-Pottier E, Boutigny H, Roussel-Delvallez M, Jourdain M, Chopin C. Effects of dental plaque antiseptic decontamination on bacterial colonization and nosocomial infections in critically ill patients. Intensive Care Med. 2000;26:1239-47.

7. Klompas M, Speck K, Howell MD, Greene LR, Berenholtz SM. Reappraisal of routine oral care with chlorhexidine gluconate for patients receiving mechanical ventilation: systematic review and meta-analysis. JAMA Intern Med. 2014;174:751-61.

8. Moher D, Liberati A, Tetzlaff J, Altman DG, PRISMA Group. Preferred reporting items for systematic reviews and meta-analyses: the PRISMA statement. BMJ. 2009;339:b2535.

9. Schünemann H, Brożek J, Guyatt G, Oxman A, eds. The GRADE handbook. Available at: http://gdt.guidelinedevelopment.org/app/handbook/handbook.html. Accessed August 10, 2018.

10. Blackstone EH, Weisel RD. The conclusion of papers published in the journal should be supported by an appropriate statistical analysis. J Thorac Cardiovasc Surg. 2014;148:2479.

11. Huebner M, Vach W, le Cessie S. A systematic approach to initial data analysis is good research practice. J Thorac Cardiovasc Surg. 2016;151:25-7.

12. Rajeswaran J, Blackstone EH. Patient-reported outcomes and importance of their appropriate statistical analyses. J Thorac Cardiovasc Surg. 2015;150:461-2.

13. DeRiso AJ II, Ladowski JS, Dillon TA, Justice JW, Peterson AC. Chlorhexidine gluconate $0.12 \%$ oral rinse reduces the incidence of total nosocomial respiratory infection and nonprophylactic systemic antibiotic use in patients undergoing heart surgery. Chest. 1996;109:1556-61.

14. Houston S, Hougland P, Anderson JJ, LaRocco M, Kennedy V, Gentry LO. Effectiveness of $0.12 \%$ chlorhexidine gluconate oral rinse in reducing prevalence of nosocomial pneumonia in patients undergoing heart surgery. Am J Crit Care. 2002; 11:567-70.
15. Lin YJ, Xu L, Huang XZ, Jiang F, Li SL, Lin F, et al. Reduced occurrence of ventilator-associated pneumonia after cardiac surgery using preoperative $0.2 \%$ chlorhexidine oral rinse: results from a single-centre single-blinded randomized trial. J Hosp Infect. 2015;91:362-6.

16. Nicolosi LN, del Carmen Rubio M, Martinez CD, Gonzalez NN, Cruz ME. Effect of oral hygiene and $0.12 \%$ chlorhexidine gluconate oral rinse in preventing ventilator-associated pneumonia after cardiovascular surgery. Respir Care. 2014:59:504-9.

17. Segers P, Speekenbrink RG, Ubbink DT, van Ogtrop ML, de Mol BA. Prevention of nosocomial infection in cardiac surgery by decontamination of the nasopharynx and oropharynx with chlorhexidine gluconate: a randomized controlled trial. JAMA. 2006;296:2460-6.

18. Rosbolt MB, Sterling ES, Fahy BG. The utility of the clinical pulmonary infec tion score. J Intensive Care Med. 2009;24:26-34.

19. Tablan OC, Anderson LJ, Besser R, Bridges C, Hajjeh R, CDC; Healthcare Infection Control Practices Advisory Committee. Guidelines for preventing healthcare-associated pneumonia, 2003: Recommendations of cdc and the healthcare infection control practices advisory committee. MMWR Recomm Rep. 2004; 53:1-36.

20. Higgins JP, Altman DG, Gotzsche PC, Juni P, Moher D, Oxman AD, et al. Cochrane Statistical Methods Group. The Cochrane Collaboration's tool for assessing risk of bias in randomised trials. BMJ. 2011;343:d5928.

21. Chan EY, Ruest A, Meade MO, Cook DJ. Oral decontamination for prevention of pneumonia in mechanically ventilated adults: systematic review and metaanalysis. BMJ. 2007;334:889.

22. Feider LL, Mitchell P, Bridges E. Oral care practices for orally intubated critically ill adults. Am J Crit Care. 2010;19:175-83.

23. Krein SL, Kowalski CP, Hofer TP, Saint S. Preventing hospital-acquired infections: a national survey of practices reported by U.S. hospitals in 2005 and 2009. J Gen Intern Med. 2012;27:773-9.

24. Hua F, Xie H, Worthington HV, Furness S, Zhang Q, Li C. Oral hygiene care for critically ill patients to prevent ventilator-associated pneumonia. Cochrane Database Syst Rev. 2016;10:CD008367.

Key Words: preoperative, chlorhexidine, mouthwash, postoperative pneumonia, cardiac surgery

Readers who found these articles interesting may also like to read the following papers found in recent and future issues of our sister publications, Seminars in Thoracic and Cardiovascular Surgery and Operative Techniques in Thoracic and Cardiovascular Surgery!

Adult: Perioperative Management

The Effect of Preoperative Anemia on Patients Undergoing Cardiac Surgery: A Propensity-Matched Analysis. Jabagi H, Boodhwani M, Tran DT, et al. Semin Thorac Cardiovasc Surg. 2019 Summer;31(2):157-163

Commentary: Preoperative Anemic Patients Have Poor Outcomes; How Can We Optimize These Patients Prior to Surgery? Greenhouse DG, Schwann TA, Engelman DT. Semin Thorac Cardiovasc Surg. 2019 Summer;31(2):164-165. 